Polish Journal of Microbiology

2012, Vol. 61, No 4, 263-271

ORGINAL PAPER

\title{
Edwardsiella ictaluri LuxS: Activity, Expression, and Involvement in Pathogenicity
}

\author{
MIN ZHANG and LI SUN* \\ Key Laboratory of Experimental Marine Biology, Institute of Oceanology, \\ Chinese Academy of Sciences, China
}

Received 21 May 2012, revised 9 September 2012, accepted 10 September 2012

\author{
Abstract
}

\begin{abstract}
Edwardsiella ictaluri is a Gram-negative bacterium and the causative agent of enteric septicemia of catfish. In this study, we examined the expression and function of the LuxS from a pathogenic E. ictaluri strain, J901. J901 was found to produce autoinducer 2 (AI-2) activity that maximized at mid-logarithmic phase and was enhanced by glucose and repressed by high temperature. Consistently, a luxS gene $\left(\right.$ luxS $\left._{E}\right)$ was identified in J901, whose expression was regulated by cell density, glucose, and temperature in a manner similar to that observed with AI-2 activity. Further analysis showed that $\operatorname{LuxS}_{\mathrm{Ei}}$ is a biologically active AI-2 synthase that was able to complement the luxS-defective phenotype of Escherichia coli DH5 $\alpha$. To examine the functional importance of LuxS, a genetically modified variant of J901, J901Ri, was constructed, in which luxS $S_{E i}$ expression was blocked by RNA interference. Compared to the wild type, J901Ri was (i) reduced in AI-2 activity to a level of 59\% of that of the wild type; (ii) impaired in both planktonic and biofilm growth; (iii) significantly attenuated in the ability to infect cultured fish cells and to cause mortality in infected fish; (iv) unable to induce the expression of certain virulence-associated genes. Addition of exogenous AI-2 failed to rescue the growth defect of J901Ri as free-living cells but restored biofilm production and the expression of virulence genes to levels comparable to those of the wild type. Taken together, these results indicate that $\mathrm{LuxS}_{\mathrm{Ei}}$ is a functional AI-2 synthase that is required for optimal cellular growth and host infection.
\end{abstract}

Ke y words: Edwardsiella ictaluri, AI-2 activity, RNA interference, quorum sensing, virulence

\section{Introduction}

Edwardsiella ictaluri is a Gram-negative, facultatively anaerobic bacterium belonging to the family Enterobacteriaceae. It is the etiological agent of enteric septicemia of catfish, which has caused severe economic losses to catfish farming industries worldwide (Wagner et al., 2002). Entry of E. ictaluri into host fish appears to be through the skin, intestinal tract, and gills (Menanteau-Ledouble et al., 2011; Morrison and Plumb 1994); once inside the host, E. ictaluri is capable of intracellular replication by resisting the killing effect of phagocytes (Booth et al., 2006). To date, several virulence factors of $E$. ictaluri have been identified, notably the secreted enzyme chondroitinase (Cooper et al., 1996), O polysaccharide (Lawrence et al., 2003; Lawrence et al., 2001), type III secretion system (T3SS) (Thune et al., 2007), hemolysin (Williams and Lawrence 2005), urease (Booth et al., 2009), regulator of sigma $E$ and a glycine cleavage system protein (Karsi etal., 2009), among them, T3SS encodes various subsets of proteins involved in different aspects of pathogenesis, this system is used by many bacteria to deliver virulence factors into host cells to subvert normal cell functions. However, the fundamental infection mechanism of E. ictaluri is not clear.

Quorum sensing is a signaling process whereby bacteria coordinate cellular behaviors according to environmental cues such as cell densities of local bacterial populations (Gonzalez and Keshavan 2006). In Gram-negative bacteria, quorum sensing is mediated by several types of signaling molecules, which include $\mathrm{N}$-Acyl homoserine lactones and autoinducer-2 (AI-2) (Miller and Bassler 2001). In Vibrio harveyi, AI-2 is a furanosyl borate diester that is synthesized from S-adenosylmethionine (SAM) through three enzymatic steps: first SAM serves as methyl donor for the production of S-adenosylhomocysteine (SAH), which is converted by the nucleosidase Pfs to S-ribosylhomocysteine (SRH); SRH is then converted by LuxS to 4,5-dihydroxy-2,3-pentanedione (DPD), from which AI-2 is derived (De Keersmaecker et al., 2006; Schauder

* Corresponding author: Institute of Oceanology, Chinese Academy of Sciences, 7 Nanhai Road, Qingdao 266071, China; phone: 86-532-82898829; fax: 86-532-82898829; email: 1sun@qdio.ac.cn 
et al., 2001). AI-2 and LuxS are widespread in diverse bacteria, and the AI- 2 molecule produced by one bacterial species can be sensed and responded to by different bacterial species, probably because the basic chemical structure of AI-2 is highly conserved (Surette and Bassler 1999; Waters and Bonnie 2005). For this reason, AI-2 has been called an interspecies communication signal. Accumulating evidences have indicated that LuxS/AI-2-mediated quorum sensing regulates many aspects of bacterial growth and infection, notably biofilm formation, motility, antibiotic susceptibility, and virulence development (Ahmed et al., 2007; Coulthurst et al., 2004; Coulthurst et al., 2007; Gonzalez Barrios etal., 2006; Herzberg et al., 2006; Kong et al., 2006; Parsek and Greenberg 2005; Rickard et al., 2006). In addition to participating in quorum sensing, LuxS is also involved in the activated methyl cycle by recycling $\mathrm{SAH}$ to homocysteine and thus contributes directly to metabolism (De Keersmaecker et al., 2006; Mc Nab et al., 2003; Vendeville et al., 2005).

Recently, the genome sequence of E. ictaluri 93-146 has been completed, which reveals the existence of a luxS homolog (Williams et al., 2012). However, the function of E. ictaluri LuxS is not known. In this study, we cloned and analyzed the $\operatorname{lu} x S$ gene, $\operatorname{lu} x S_{E i}$, from a pathogenic E. ictaluri strain J901. We found that Lux$\mathrm{S}_{\mathrm{Ei}}$ likely plays a role in both cellular metabolism and pathogenicity, the latter via AI-2-mediated quorum sensing system.

\section{Experimental}

Materials and Methods

Bacterial strains and growth conditions. Edwardsiella ictaluri J901, a pathogenic strain isolated from catfish, was purchased from Institute of Hydrobiology, Chinese Academy of Sciences (Wuhan, China). Escherichia coli DH5a was purchase from Takara (Dalian, China). Vibrio harveyi BB120 and BB170 were purchased from American Type Culture Collection (ATCC, USA). All strains were cultured in Luria-Bertani (LB) medium at $37^{\circ} \mathrm{C}$ (for E. coli) or $28^{\circ} \mathrm{C}$ (for all others). Where indicated, glucose was added at a final concentration of $0.5 \%$, and ampicillin and polymyxin B were added at $100 \mu \mathrm{g} / \mathrm{ml}$ and $100 \mu \mathrm{g} / \mathrm{ml}$ respectively. The mean generation time $(g)$ of bacterial growth was calculated as described by Eagon (1962).

AI-2 assay. AI-2 assay was performed according to Surette and Bassler (1999). To prepare cell-free culture supernatant, bacterial cells were grown in LB medium at $28^{\circ} \mathrm{C}$ for overnight, and the culture was diluted $1: 100$ in fresh LB medium. Two milliliters of cell culture were taken every 30 minutes, and the cell-free super- natant was obtained by centrifugation and then filtering through a $0.22-\mu \mathrm{m}$ filter (Millipore, Billerica, MA, USA). For measurement of bioluminescence induction, overnight culture of the $V$. harveyi strain $\mathrm{BB} 170$ grown in $\mathrm{AB}$ medium at $28^{\circ} \mathrm{C}$ was diluted 1:5000 in fresh $\mathrm{AB}$ medium supplemented with cell-free culture fluids $(10 \%)$ of the tested strains or with the growth medium (as the control). The growth was continued and light production was measured by using a Glomax luminometer (Promega, Madison, WI, USA).

Cloning of $\operatorname{luxS} S_{E i}$. The primers used in this study are

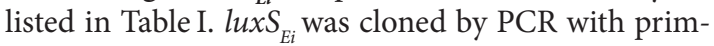
ers F5/R6 designed according to the luxS sequence of E. ictaluri 93-146 (GenBank accession no. CP001600). The sequence of $l u x S_{E i}$ has been deposited in GenBank database under the accession number JQ272177.

Plasmid and strain constructions. pBTEiS, which expresses $\operatorname{lu} x S_{E i}$, was constructed as follows. $\operatorname{lu} x S_{E i}$ was amplified by PCR with primers F5/R6, and the PCR products were ligated into the TA cloning vector PEASY-T1 (Transgen, China) resulting in pESEiS, which was digested with EcoRV, and the $\operatorname{luxS} S_{E i}$-containing fragment was inserted into pBT (Zhang et al., 2008) at the SmaI site, resulting in pBTEiS. To construct pJRSi, which expresses antisense $\operatorname{luxS_{Ei}}$ under the trc promoter, the antisense strand of $\operatorname{luxS} S_{E i}$ was amplified with primers $\mathrm{F} 5 / \mathrm{R} 5$, and the PCR products were ligated into $\mathrm{pBT}$ as above, resulting in $\mathrm{pBTSi}$, which was digested with SwaI, and the fragment containing the $\mathrm{P}_{t r c}-l u x \mathrm{~S}_{E i}$ antisense RNA was inserted into the EcoRV site of pJRA (Zhang et al., 2008), resulting in pJRSi. pJRSi and the control vector pJRA were introduced separately into J901 via conjugation as described previously (Sun et al., 2009), and the transformants were named J901Ri and J901C respectively. J901C was used as control of J901Ri.

Quantitative real time reverse transcriptase-PCR (qRT-PCR). qRT-PCR was carried out in an Eppendorf Mastercycler (Eppendorf, Hamburg, Germany) using the SYBR ExScript qRT-PCR Kit (Takara, Dalian, China) as described previously (Zheng et al., 2010). PCR efficiency (99.9\%) was determined as described previously (Zheng and Sun 2011). Melting curve analysis of amplification products was performed at the end of each PCR to confirm that only one PCR product was amplified and detected. The expression level of the taget gene was analyzed using comparative threshold cycle method $\left(2^{-\triangle \triangle C T}\right)$ with $16 \mathrm{~S}$ rRNA as the control. The PCR primers for 16S rRNA gene (16SF/16SR), lux $\mathrm{S}_{E i}$ (LuxSF4/ LuxSR4), and the virulence-associated genes are listed in Table I.

Complementation by exogenous AI-2. J901 was cultured in LB medium to $\mathrm{OD}_{600}$ of 0.85 , and cell-free culture supernatant (prepared as above) was added at a final concentration of $10 \%$ to the growth medium of bacterial cells under various examination. 
Table I

PCR primers used in this study

\begin{tabular}{|c|c|c|}
\hline Primers & ${ }^{a}$ Sequences $\left(5^{\prime} \rightarrow 3^{\prime}\right)$ & Target genes \\
\hline $16 \mathrm{SF}$ & CAACGAGCGCAACCCTTATC & 16S rRNA \\
\hline 16SR & CGTGTGTAGCCCTACTCGTA & \\
\hline EihAF & GGCGTTGGTGGTGGGTAA & $e i h A$ \\
\hline EihAR & CCCCTCCCGCACCTATCT & \\
\hline EseBF & TTGAAGTGATGTCTGCAAAGGC & $e s e B$ \\
\hline EseBR & TTGAGTTGAGCGATGATGGAAT & \\
\hline EseDF & GCTGTCGCTCAAATCGTCG & eseD \\
\hline EseDR & CGGGTCAGATCATTGGCATAG & \\
\hline EsrAF & CGATACCCCGCAGCACAT & esrA \\
\hline EsrAR & CTTCGCCGCAATCACCC & \\
\hline EvpAF & CATTCCGCACTGAAAATACCC & $\operatorname{evp} A$ \\
\hline EvpAR & TCAGGAGTGAAATCCGCCATA & \\
\hline EvpBF & GCCGACCTGGGATTTATCG & $\operatorname{evp} B$ \\
\hline EvpBR & CCTGGCGTTGGCATTAGC & \\
\hline LuxSF4 & AAGGCGGCGATGGAGGA & $\operatorname{luxS}$ \\
\hline LuxSR4 & TGGCGGGCAATCTGGT & \\
\hline LuxSF5 & GATATCGCAATATGGGAAAAGGT (EcoRV) & $\operatorname{luxS}$ \\
\hline LuxSR5 & GATATCCGGCAGAATGGCAT (EcoRV) & \\
\hline LuxSR6 & $\underline{\text { GATATCTAGGGGTGCAGATGGG (EcoRV) }}$ & \\
\hline Orf26F & CAGGTGGCGGGAAAGCA & orf26 \\
\hline Orf26R & ATACCGAATCATAACGATAGGAGG & \\
\hline WbiTF & GTGTATAACCTGGGGACGGG & wbiT \\
\hline WbiTR & CAAGGCGGGATTGGACC & \\
\hline
\end{tabular}

${ }^{a}$ Underlined nucleotides are restriction sites of the enzymes indicated in parentheses.

Biofilm production analysis. Biofilm formation on a polystyrene surface was determined exactly as described by Xu et al., (2006). Briefly, cells were cultured in LB medium to exponential phase and diluted 1:100 into fresh LB. The diluted cultures were transferred into a 96-well polystyrene plate. After incubating at $28^{\circ} \mathrm{C}$ for $24 \mathrm{~h}$, the plate was washed with PBS and the attached cells were treated with Bouin fixative and stained with $1 \%$ crystal violet. The unbound dye was removed by rinsing the plate several times with running water. The bound dye was eluted in ethanol, and the eluates were measured for absorbance at $A_{570}$.

Infection of zebrafish. J901Ri and J901C were cultured to an $\mathrm{OD}_{600}$ of 0.8 in LB medium, washed with $\mathrm{PBS}$, and resuspended in PBS to $1 \times 10^{8} \mathrm{CFU} / \mathrm{ml}$. Zebrafish $(0.3 \pm 0.05 \mathrm{~g})$ were purchased from Nanshan Market (Qingdao, China) and maintained at $25^{\circ} \mathrm{C}$ in aerated freshwater that was changed daily. Fish were divided randomly into two groups $(\mathrm{N}=30)$ and anaesthetised by immersion in a $100 \mu \mathrm{g} \mathrm{ml}^{-1}$ solution of tricaine methane sulfonate (MS-222, Sigma, St Louis, MO, USA) before injection. The fish were injected intraperitoneally (i.p.) with $10 \mu \mathrm{J}$ J901Ri or J901C and monitored for mortality for 15 days post-infection. The animal experiments were conducted in accordance with the "Regulations for the Administration of Affairs Concerning Experimental Animals" promulgated by the State Science and Technology Commission of Shandong Province.

Infection of ZF4 cells. Infection of ZF4 cells was performed as follows. J901Ri and J901C were cultured in $\mathrm{LB}$ medium to an $\mathrm{OD}_{600}$ of 0.80 , washed with $\mathrm{PBS}$, and resuspended to $1 \times 10^{6} \mathrm{CFU} / \mathrm{ml}$ in DMEM-F12 medium (Thermo Scientific HyClone, Beijing, China) supplemented with $10 \%$ fetal calf serum. ZF4 cells were purchased from ATCC and maintained at $28^{\circ} \mathrm{C}$ in 96-well culture plates containing DMEM-F12 medium supplemented with $10 \%$ fetal calf serum, $100 \mathrm{IU} / \mathrm{ml}$ penicillin, and $100 \mu \mathrm{g} / \mathrm{ml}$ streptomycin according to ATCC recommendations. The cells were grown to monolayer and added with J901Ri or J901C (100 $\mu \mathrm{l} /$ well). The plates were incubated at $28^{\circ} \mathrm{C}$ for $0.5 \mathrm{~h}$, followed by washing $3 \times$ with PBS to remove unattached bacteria. To determine the number of bacterial cells associated with ZF4 cells, the washed ZF4 cells were lysed with $1 \%$ Triton X-100, and the lysates were diluted in LB medium and plated in triplicate on LB agar plates supplemented with ampicillin and polymyxin B. After 


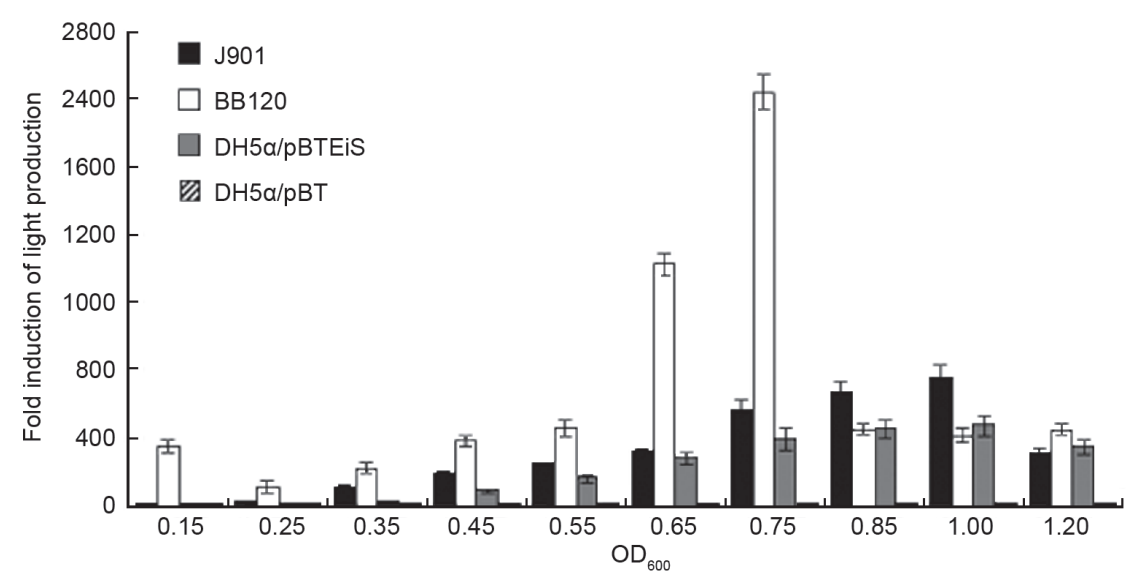

Fig. 1. AI-2 activity detection.

Cell-free culture supernatants of Edward ictaluri J901, DH5a/pBTEiS, DH5a/pBT, and Vibrio harveyi BB120 were taken at various growth points and assayed for AI-2 activity by determining the ability of the supernatant to stimulate light production in BB170. Data are presented as means \pm SE $(\mathrm{N}=3){ }^{* *}, \mathrm{P}<0.01$

incubation at $28^{\circ} \mathrm{C}$ for $32 \mathrm{~h}$, the colonies that appeared on the plates were counted. The genetic nature of the colonies was verified by PCR and subsequent sequence analysis of selected PCR products. Each assay was performed in triplicate.

Statistical analysis. All statistical analyses were performed with SPSS 15.0 software (SPSS Inc., Chicago, IL, USA). Data were analyzed with analysis of variance (ANOVA) and expressed as means \pm SE. In all cases, statistical significance was defined as $P<0.05$.

\section{Results}

Regulated production of AI-2 in E. ictaluri J901. To examine whether E. ictaluri J901 produced active AI-2, the strain was cultured in LB medium to different growth phases and examined for AI-2 activity by measuring the ability of the cell-free culture supernatant to stimulate bioluminescence in $\mathrm{BB} 170$, a reporter strain that is defective in AI-1 response but able to respond to AI-2 (Surette and Bassler 1999). The results showed that the AI-2 activity, as a reflection of stimulated bioluminescence production in the reporter strain, of J901 increased with cell density until $\mathrm{OD}_{600}$ of 1.0, where peak activity was reached, and then declined at $\mathrm{OD}_{600}$ of 1.2 (Fig. 1). However, at each of the examined point, the amount of light production induced by the culture supernatant of J901 was much lower than that induced by the supernatant of BB120, which is the parental strain of $\mathrm{BB} 170$. Compared to growth in standard $\mathrm{LB}$ medium at $28^{\circ} \mathrm{C}$, growth in $\mathrm{LB}$ medium at $37^{\circ} \mathrm{C}$ decreased the AI-2 activity of J901 by 139 fold, whereas growth in LB medium supplemented with glucose increased AI-2 activity by 2.4 fold (Fig. 2).
J901 possesses a luxS gene that encodes an active AI-2 Synthase. The above results suggested the existence in J901 of a luxS gene, which was subsequently cloned and named $\operatorname{lu} x S_{E i}$. Sequence analysis showed that $l u x S_{E i}$ encodes a protein of 171 amino acid residues that differs in two residues from the reported LuxS of E. ictaluri 93-146 (GenBank accession no. ACR70372.1) and shared 94\% identity to the LuxS of Edwardsiella tarda. Since AI-2, the product of LuxS, is known to be an interspecies signaling molecule and able to function in a cross-species manner in many bacteria, we examined the activity of $\operatorname{LuxS}_{\mathrm{Ei}}$ by determining its ability to complement $\mathrm{DH} 5 \mathrm{a}$, which lacks a functional luxS gene and consequently exhibits an AI2-defective phenotype (Surette and Bassler 1999). For this purpose, the plasmid pBTEiS, which expresses lux$S_{E i}$, was constructed. E. coli DH5a was transformed with

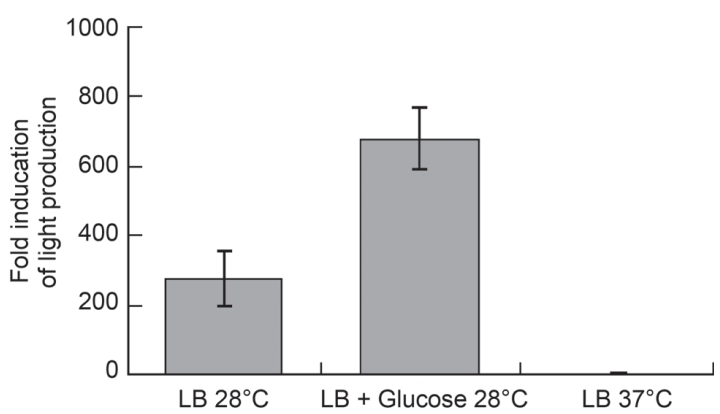

Fig. 2. AI-2 activity of Edward ictaluri J901 under different growth conditions.

J901 was assayed for AI-2 activity after culturing in LB medium to $\mathrm{OD}_{600} \sim 0.55$ under various conditions, i.e., with or without $0.5 \%$ glucose, at $37^{\circ} \mathrm{C}$, or at $28^{\circ} \mathrm{C}$ (control). AI-2 activity is presented as fold induction of light production over the control. Data are presented as the means $\pm \mathrm{SE}(\mathrm{N}=3) .{ }^{* *}, \mathrm{P}<0.01$. 
pBTEiS and the control plasmid pBT (the backbone plasmid of pBTEiS) respectively. The resulting transformants, DH5a/pBTEiS and DH5a/pBT respectively, were cultured to different cell densities, and the culture supernatants were examined for AI- 2 activity. The results showed that the supernatant of DH5a/pBTEiS exhibited AI-2 activity, though much lower than that of BB120, in a manner that increased with cell density and reached maximum at $\mathrm{OD}_{600}$ of 0.85 (Fig. 1). In contrast, no AI-2 activity was detected in the supernatant of $\mathrm{DH} 5 \alpha / \mathrm{pBT}$. These results indicate that $\mathrm{LuxS}_{\mathrm{Ei}}$ adds functionality to luxS lacking DH5a.

Expression of $l u x S_{E i}$ is regulated by growth phase and growth condition. Since, as shown above, AI-2 activity in $\mathrm{J} 901$ was regulated by growth phase and growth condition, we examined whether $\operatorname{lux} S_{E i}$ expression was similarly regulated. For this purpose, J901 was cultured to $\mathrm{OD}_{600}$ of $0.2,0.4,0.6,0.8,1.0$, and 1.5 respectively, and $\operatorname{lu} x S_{E i}$ expression was determined by qRT-PCR. The results showed that $\operatorname{lux} S_{E i}$ expression increased with cell density and reached maximum at $\mathrm{OD}_{600}$ of 0.8 , after which point $\operatorname{luxS} S_{E i}$ expression dropped to levels comparable to that at $\mathrm{OD}_{600}$ of 0.2 (Fig. 3A). Compared to J901 cultured in standard LB medium at $28^{\circ} \mathrm{C}$, J901 cultured in LB medium containing glucose exhibited significantly increased $l u x S_{E i}$ expression, whereas J901 cultured in LB medium at $37^{\circ} \mathrm{C}$ exhibited significantly decreased $l u x S_{E i}$ expression (Fig. 3B). These results, together with those that showed regulated $\mathrm{AI}-2$ production as observed above, indicate a correlation between $l u x S_{E i}$ expression and AI-2 activity.

Effect of interference with $l u x S_{E i}$ expression. (i) Effect on AI-2 activity. To examine the functional importance of LuxS ${ }_{\mathrm{Ei}}$, we constructed a J901 variant, J901Ri, in which $\operatorname{lux} S_{E i}$ expression was interfered by antisense RNA. We also tried but failed to create a lux$S_{E i}$-defective mutant via targeted mutation of the $l u x S_{E i}$ gene, which suggests a possibility of LuxS being essential to the fundamental physiology of J901. qRT-PCR analysis showed that when the cells were cultured in LB medium at $\mathrm{OD}_{600}$ of 0.8 , which, as shown above, was the point where peak induction of $l u x S_{E i}$ was observed, $\operatorname{luxS_{Ei}}$ expression in J901Ri was significantly $(P<0.01)$ decreased to a level that was approximately $13 \%$ of that in the control strain J901C or in the wild type J901, the latter two exhibited comparable levels of $l u x S_{E i}$ expression. These results indicate that, as designed, $l u x S_{E i}$ expression in J901Ri was markedly reduced. Subsequent AI-2 assay showed that the AI-2 activity of J901Ri was approximately $41 \%$ lower than that of J901C. Hence, interfering with $l u x S_{E i}$ expression resulted in reduced AI-2 production.

(ii) Effect on growth. Growth analysis showed that when cultured in standard LB medium and in irondepleted LB medium caused by the presence of $50 \mu \mathrm{M}$
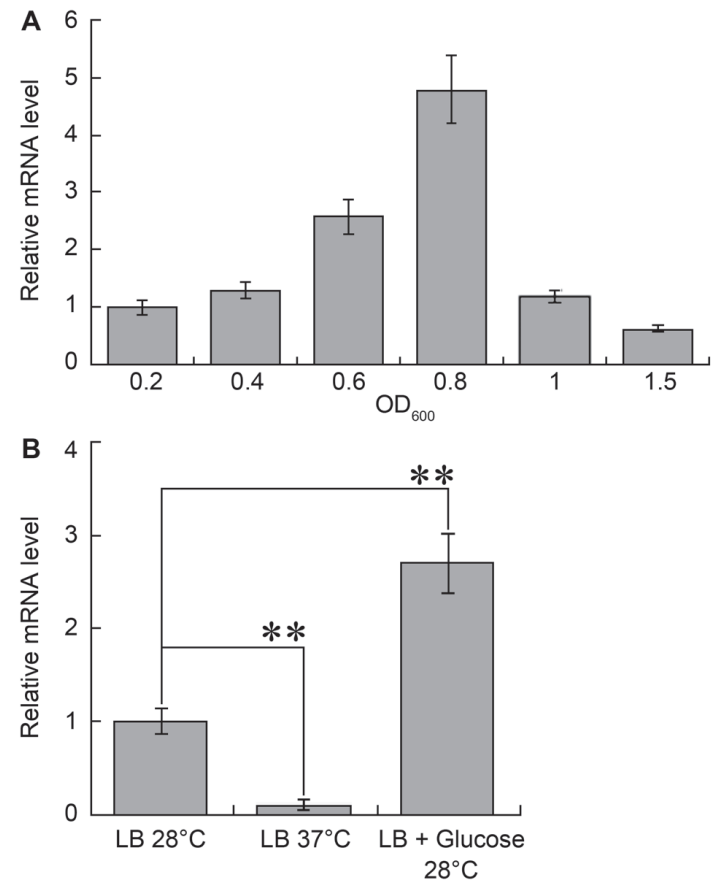

Fig. 3. Expression of $\operatorname{lux} S_{E i}$ at different growth phases (A) and under different growth conditions (B).

(A) $l u x S_{E i}$ expression in Edward ictaluri J901 was determined by quantitative real time RT-PCR (qRT-PCR) after the cells having been grown to different cell densities. (B) $l u x S_{E i}$ expression in J901 was determined by qRT-PCR after the cells having been grown in $\mathrm{LB}$ medium to $\mathrm{OD}_{600} 0.55$ under various conditions, i.e., with or without $0.5 \%$ glucose, at $37 \mathrm{c}$, or at $28^{\circ} \mathrm{C}$ (control).

Data are presented as means $\pm \mathrm{SE}(\mathrm{N}=3) .{ }^{*}, \mathrm{P}<0.01$.

iron-chelator 2,2'-dipyridyl, the mean generation time $(\mathrm{g})$ values of J901Ri were $150 \mathrm{~min}$ and $222 \mathrm{~min}$ respectively, while the $g$ values of J901C were $132 \mathrm{~min}$ and 169 min respectively under these conditions (Fig. 4A). Likewise, biofilm growth analysis showed that compared to J901C, J901Ri exhibited significantly reduced ability to form biofilm on polystyrene surface (Fig. 4B). To examine whether the growth defect was caused by interfered quorum sensing, AI-2-containing culture supernatant was added to J901Ri. The results showed that the presence of exogenous AI-2 failed to restore the growth of J901Ri in LB medium but rescued the defect of J901Ri in biofilm production (Fig. 4B and data not shown), suggesting that AI-2-mediated signaling is required for biofilm growth but not for planktonic growth.

Effect on infection of zebrafish and ZF4 cells. To examine whether reduced $l u x S_{E i}$ expression had any effect on virulence, the infectivity of J901Ri was determined using both live zebrafish and cultured zebrafish cells. For live fish infection, zebrafish were infected via i.p. injection with the same dose of J901Ri or J901C and monitored for mortality. The results showed that mortality began to occur at 3 days post-infection in 

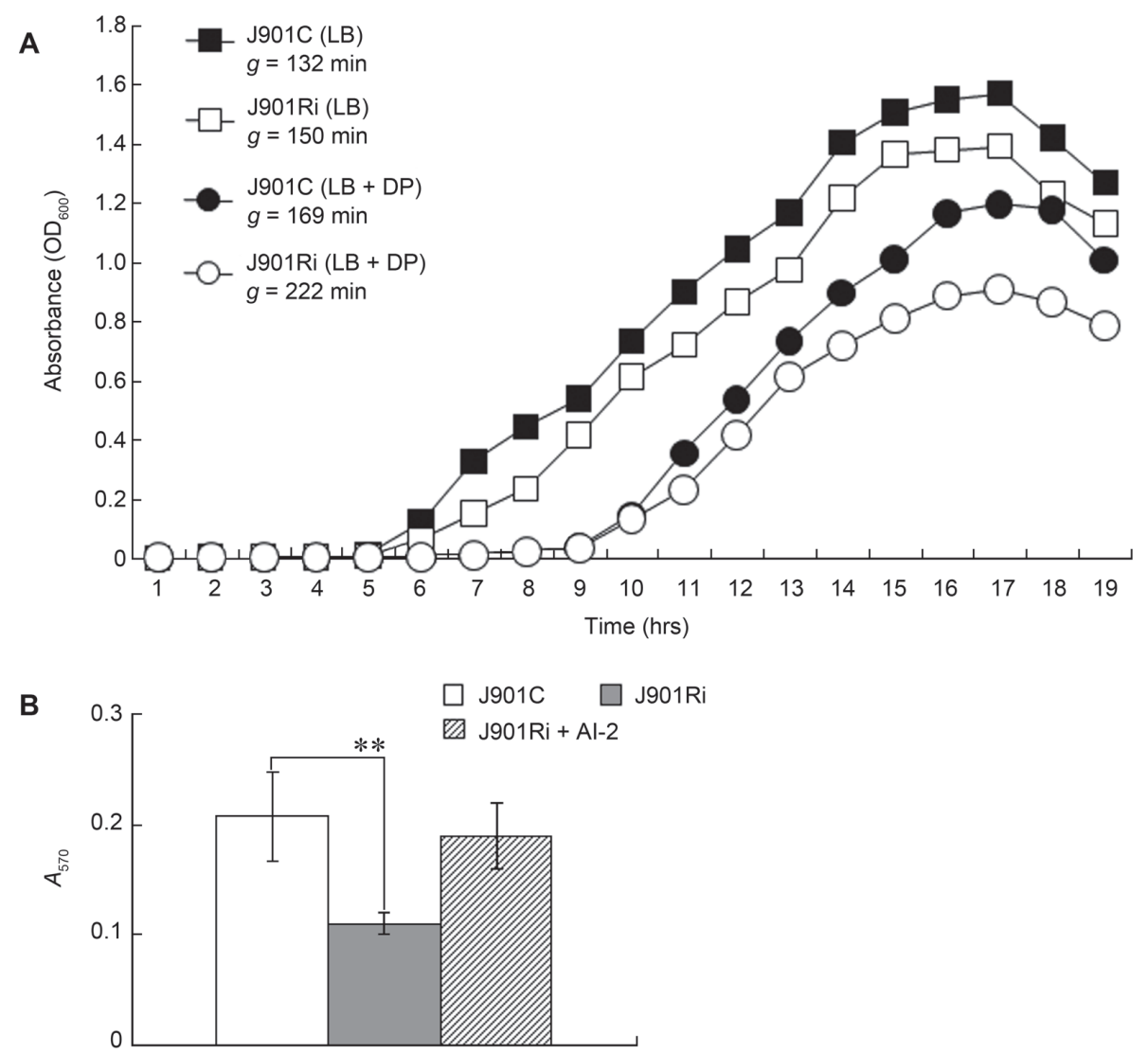

Fig. 4. Growth (A) and biofilm production (B) of J901Ri and J901C under different conditions.

(A) Cells were cultured in LB medium with or without $50 \mu \mathrm{M}$ 2,2'-dipyridyl (DP) and monitored for growth. $g$, mean generation time. (B) J901Ri and J901C were grown in polystyrene plates for $24 \mathrm{~h}$ in the presence or absence of exogenous AI- 2 and then assayed for biofilm production. Data are presented as the means $\pm \mathrm{SE}(\mathrm{N}=4)$. ${ }^{* *}, \mathrm{P}<0.01$.

both J901Ri- and J901C-infected fish, however, J901Cinfected fish reached $100 \%$ accumulative mortality by 5 days post-infection, while J901Ri-infected fish exhibited an accumulative mortality rate of $40 \%$ (Fig. 5). To examine the infectivity of J901Ri at cellular level, ZF4 cells (a cultured zebrafish cell line) were treated with J901Ri or J901C for $0.5 \mathrm{~h}$, and cellular infection was subsequently examined by bacterial recovery analysis, which determined the number of J901Ri or J901C that had succeeded in adherence to or/and penetration into ZF4 cells. The results showed that the bacterial recovery from J901Ri-infected cells was 2.3-fold less than that from J901C-infected cells.

Effect on the expression of virulence-associated genes. Since the above results indicated an involvement of LuxS $_{\mathrm{Ei}}$ in host infection, we examined whether interfering with $\operatorname{lux} S_{E i}$ expression had any effect on the expression of virulence genes. For this purpose, qRTPCR was carried out to determine the expression of the type three secretion system genes orf26, esr A, eseB, and eseD, the type six secretion system genes evpA and $\operatorname{evp} B$, the haemolysin gene eihA, and the wbiT gene involved in $\mathrm{O}$ polysaccharide biosynthesis. The results showed that except for $\operatorname{evp} A$ and $\operatorname{evp} B$, which exhibited comparable levels of expression in J901Ri and J901C, the expressions of all other genes were signifi-

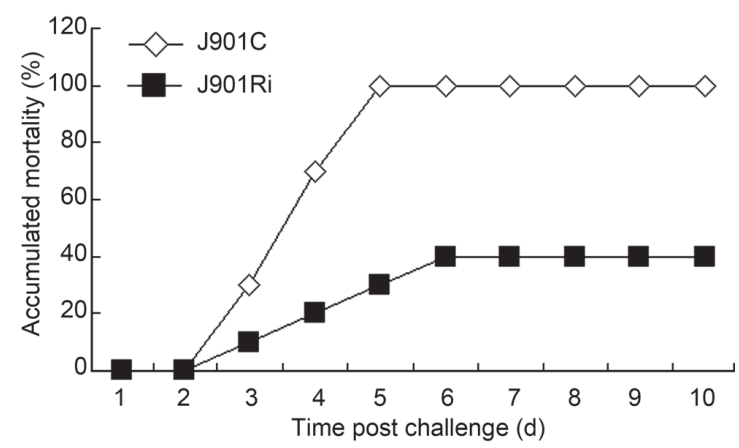

Fig. 5. Cumulative mortality curves induced by J901Ri and J901C. Zebrafish were infected with J901Ri and J901C respectively via i.p. injection and monitored daily for mortality. 


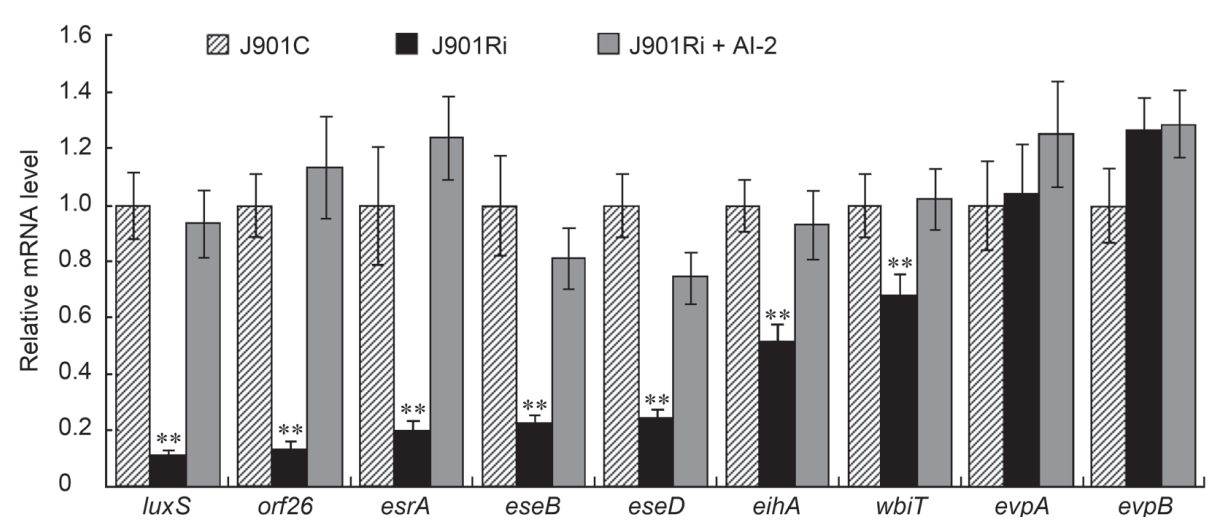

Fig. 6. Expression of virulence genes in J901Ri and J901C.

Total RNA was extracted from J901Ri and J901C grown to $\mathrm{OD}_{600} \sim 0.85$ in the presence or absence of exogenous AI- 2 and used for quantitative real time RT-PCR analysis of virulence-associated genes. For each gene, the expression level of J901C was set as 1 . Data are presented as the means \pm SE $(\mathrm{N}=4)$. ${ }^{* *}, \mathrm{P}<0.01$.

cantly reduced in J901Ri (Fig. 6). To examine whether the above observed effect on gene expression was due to reduced AI-2 production in J901Ri, exogenous AI-2 was added to the cell culture of J901Ri. Subsequent qRT-PCR analysis showed that addition of AI-2 restored the expression of all the genes in J901Ri to levels similar to those in J901C (Fig. 6).

\section{Discussion}

In this study, we examined the expression, activity, and biological function of the luxS gene from a pathogenic E. ictaluri isolate, J901. J901 was found to exhibit AI-2 activity that increased with cell density and reached maximum at $\mathrm{OD}_{600}$ of 1.0. These results suggest that $\mathrm{J} 901$ produced active AI- 2 in a manner that was regulated by cell density, which is consistent with the role of AI-2 as a quorum sensing signal. The observation that the AI-2 activity of J901 was much lower than that of BB120 suggests that J901 may either produce less amount of AI-2 than BB120 under the culture condition or that the AI-2 of J901 is less effective in triggering the signaling process of quorum sensing that leads to light production in BB170 due to, for example, certain structural difference between the AI-2 molecules of J901 and BB120. Previous studies have shown that in bacteria such as Salmonella typhimurium and Edwardsiella tarda, AI-2 production is affected by various environmental stimuli including $\mathrm{pH}$, osmolarity, and temperature (Surette et al., 1999; Zhang et al., 2008). It is known that in E.coli, AI-2 synthesis and uptake are regulated by catabolite repression through the cyclic AMP-CRP complex that indirectly represses luxS expression (Taga et al., 2003; Wang et al., 2005; Xavier and Bassler 2005). In our study, we found that compared to growth in standard $\mathrm{LB}$ medium at $28^{\circ} \mathrm{C}$, growth in LB medium at $37^{\circ} \mathrm{C}$ decreased AI-2 activity, whereas growth in the presence of glucose increased AI-2 activity. These results indicate that, as observed in other bacterial species, the AI- 2 activity of E. ictaluri is regulated by carbon source and temperature.

In agreement with the presence of AI-2 activity in J901, a luxS gene, $\operatorname{lux} S_{E i}$, was identified in J901. To examine whether $\operatorname{lux} S_{E i}$ encodes a functional enzyme, the gene was sub-cloned via the plasmid $\mathrm{pBTEiS}$ into the luxS-defective E. coli DH5a. Subsequent analysis showed that the recombinant DH5a/pBTEiS acquired the ability to produce AI-2 activity, suggesting that $\mathrm{LuxS}_{\mathrm{Ei}}$ is an active enzyme that is able to catalyze the production of functional AI-2 in DH5a. qRT-PCR analysis showed that $l u x S_{E i}$ expression was regulated by cell density, glucose, and temperature in a manner similar to that observed with AI-2 activity, suggesting that the AI-2 activity of J901 was controlled at the expression level of $\operatorname{luxS} S_{E i}$. Similar observations have been made in other bacterial species such as E.coli and E.tarda (Zhang et al., 2008; Wang et al., 2005; Taga et al., 2001). In our study, the above hypothesis was supported further by the observation that J901Ri, in which $l u x S_{E i}$ expression was interfered, exhibited reduced AI-2 activity.

Growth study showed that compared to the control strain J901C, J901Ri was retarded in growth rate and impaired in the ability to form biofilm, which indicate a requirement of LuxS $_{\mathrm{Ei}}$ for optimal growth of J901 both as free-living cells and as surface-attached cells. The observation that addition of exogenous AI-2 did not restore the growth of J901Ri in LB medium but rescued the biofilm growth of J901Ri suggests an involvement of AI-2-mediated quorum sensing in biofilm growth but not in planktonic growth. It is likely that the restored biofilm growth in J901Ri was due to the ability of the 
added AI-2 to activate the quorum sensing pathway, which in turn activated the regulatory system that controls biofilm production. Given that LuxS is known to play an important metabolic function in the activated methyl cycle and that luxS mutation affects metabolic genes required for normal growth (Mc Nab et al., 2003; Doherty et al., 2006; Kendall et al., 2007; Winzer et al., 2002), our results suggest a likely participation of $\mathrm{LuxS}_{\mathrm{Ei}}$ in the central metabolic process of E. ictaluri.

Association between LuxS and pathogenicity has been reported for many bacterial species (Coulthurst et al., 2004; Coulthurst et al., 2007; Joyce et al., 2004; Novak et al., 2010; Plummer et al., 2011). In our study, we found that when zebrafish were infected with J901Ri or J901C, $40 \%$ accumulative mortality was observed in J901Ri-infected fish, whereas $100 \%$ accumulative mortality was observed in J901C-infected fish. These results indicate that interference with $\operatorname{lux} S_{E i}$ expression attenuated the overall virulence of J901. Consistently, cellular infection study showed that after $0.5 \mathrm{~h}$ of incubation with ZF4 cells, the number of cell-associated J901Ri was 2.3 -fold less than that of cell-associated J901C. Since the infection time was short, it is likely that the reduced bacterial recovery of J901Ri was due to reduced infectivity rather than reduced growth capacity.

In some pathogenic bacteria such as E. coli, V. harveyi, Streptococcus pyogenes, and E.tarda, LuxS was found to regulate the expression and production of various virulence factors including T3SS components, proteases, and exotoxins (Henke and Bassler, 2004; Lyon et al., 2001; Marouni and Sela 2003; Sircili et al., 2004). In our study, we found that interference with lux $S_{E i}$ expression significantly reduced the expression of the genes involved in T3SS, haemolysin production, and $\mathrm{O}$ polysaccharide biosynthesis. Since T3SS, O polysaccharide, and hemolysin are known to be associated with pathogenicity (Lawrence et al., 2003; Lawrence et al., 2001; Thune et al., 2007; Williams and Lawrence 2005), these results suggest that reduced expression of these genes may at least in part account for the reduced infectivity of J901Ri. The observation that the presence of exogenous AI-2 restored the expression of all these genes in J901Ri suggests that $\mathrm{LuxS}_{\mathrm{Ei}}$ regulates the expression of these virulence genes through AI-2-mediated quorum sensing.

In conclusion, the results of this study showed that E. ictaluri $\mathrm{J} 901$ exhibits regulated production of AI-2 that is controlled at the level of $\operatorname{lu} x S_{E i}$ expression, and that interference with the regulated expression of

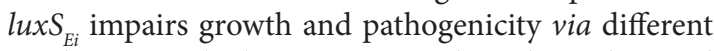
mechanisms which are, respectively, independent and dependent on quorum sensing. These results suggest a participation of LuxS $_{\mathrm{Ei}}$ in both central metabolism and AI-2-mediated signaling process, the latter may consequently affect bacterial virulence.

\section{Acknowledgements}

This work was supported by the Knowledge Innovation Program of the Chinese Academy of Sciences grants Y02420101Q and KSCX2-EW-G-12B.

\section{Literature}

Ahmed N.A., F.C. Petersen and A.A. Scheie. 2007. AI-2 quorum sensing affects antibiotic susceptibility in Streptococcus anginosus. J. Antimicrob. Chemoth. 60: 49-53.

Booth N.J., J.B. Beekman and R.L. Thune. 2009. Edwardsiella ictaluri encodes an acid-activated urease that is required for intracellular replication in channel catfish (Ictalurus punctatus) macrophages. Appl. Environ. Microb. 75: 6712-6720.

Booth N.J., A. Elkamel and R.L. Thune. 2006. Intracellular replication of Edwardsiella ictaluri in channel catfish macrophages. J. Aquat. Anim. Health 18: 101-108.

Cooper R.K., E.B. Shotts and L.K. Nolan. 1996. Use of a minitransposon to study chondroitinase activity associated with Edwardsiella ictaluri. J. Aquat. Anim. Health 8: 319-324.

Coulthurst S.J., S. Clare, T.J. Evans, I.J. Foulds, K.J. Roberts, M. Welch, G. Dougan and G.P. Salmond. 2007. Quorum sensing has an unexpected role in virulence in the model pathogen Citrobacter rodentium. EMBO. J. 8: 698-703.

Coulthurs T., S.J., C.L. Kurz and G.P.C. Salmond. 2004. luxS mutants of Serratia defective in autoinducer-2-dependent 'quorum sensing' show strain-dependent impacts on virulence and production of carbapenem and prodigiosin. Microbiology 150: 1901-1910. De Keersmaecker S.C.J., K. Sonck and J. Vanderleyden. 2006. Let LuxS speak up in AI-2 signaling. Trends Microbiol. 14: 114-119. Doherty N., M.T. Holden, S.N. Qazi, P. Williams and K. Winzer. 2006. Functional analysis of luxS in Staphylococcus aureus reveals a role in metabolism but not quorum sensing. J. Bacteriol. 188: 2885-2897.

Eagon R.G. (1962). Pseudomonas natriegens, a marine bacterium with a generation time of less than 10 minutes. J. Bacteriol. 83: 736-737.

Gonzalez Barrios A.F., R. Zuo, Y. Hashimoto, L. Yang, W.E. Bentley and T.K. Wood. 2006. Autoinducer 2 controls biofilm formation in Escherichia coli through a novel motility quorum-sensing regulator (MqsR, B3022). J. Bacteriol. 188: 305-316.

Gonzalez J.E. and N.D. Keshavan. 2006. Messing with bacterial quorum sensing. Microbiol. Mol. Biol. R. 70: 859-875.

Henke J.M. and B.L. Bassler. 2004. Quorum sensing regulates type III secretion in Vibrio harveyi and Vibrio parahaemolyticus. J. Bacteriol. 186: 3794-3805.

Herzberg M., I.K. Kaye, W. Peti and T.K. Wood. 2006. YdgG (TqsA) controls biofilm formation in Escherichia coli K-12 through autoinducer 2 transport. J. Bacteriol.188: 587-598.

Joyce E.A., A. Kawale, S. Censini, C.C. KIM, A. Covacci and S. Falkow. 2004. LuxS is required for persistent pneumococcal carriage and expression of virulence and biosynthesis genes. Infect. Immun. 72: 2964-2975.

Karsi A., N. Gülsoy, E. Corb, P.R. Dumpala and M.L. Lawrence. 2009. High-throughput bioluminescence-based mutant screening strategy for identification of bacterial virulence genes. Appl. Environ. Microb. 75: 2166-2175.

Kendall M.M., D.A. Rasko and V. Sperandio. 2007. Global effects of the cell-to-cell signaling molecules autoinducer-2, autoinducer-3, and epinephrine in a luxS mutant of enterohemorrhagic Escherichia coli. Infect. Immun. 5: 4875-4884.

Kong K.F., C. Vuong and M. Otto. 2006. Staphylococcus quorum sensing in biofilm formation and infection. Int. J. Med. Microbiol. 296: 133-139. 
Lawrence M.L., M.M. Banes, P. Azadi and B.Y. Reeks. 2003. The Edwardsiella ictaluri $\mathrm{O}$ polysaccharide biosynthesis gene cluster and the role of $\mathrm{O}$ polysaccharide in resistance to normal catfish serum and catfish neutrophils. Microbiology 149: 1409-1421.

Lawrence M.L., M.M. Banes and M.L. Williams. 2001. Phenotype and virulence of an isogenic lipopolysaccharide $\mathrm{O}$ side chain mutant strain of Edwardsiella ictaluri. J. Aquat. Anim. Health 13: 291-299. Lyon W.R., J.C. Madden, J.C. Levin, J.L. Stein and M.G. Caparon. 2001. Mutation of luxS affects growth and virulence factor expression in Streptococcus pyogenes. Mol. Microbiol. 42: 145-157.

Marouni M.J. and S. Sela. 2003. The luxS gene of Streptococcus pyogenes regulates expression of genes that affect internalization by epithelial cells. Infect. Immun. 71: 5633-5639.

Mc Nab R., S.K. Ford, A. EL-Sabaeny, B. Barbieri, G.S. Cook and R.J. Lamont. 2003. LuxS-based signaling in Streptococcus gordonii: autoinducer 2 controls carbohydrate metabolism and biofilm formation with Porphyromonas gingivalis. J. Bacteriol. 185: 274-284. Menanteau-Ledouble S., A. Karsi and M.L. Lawrence. 2011. Importance of skin abrasion as a primary site of adhesion for Edwardsiella ictaluri and impact on invasion and systematic infection in channel catfish Ictalurus punctatus. Vet. Microbiol. 148: 425-430.

Miller M.B. and B.L. Bassler. 2001. Quorum sensing in bacteria. Annu. Rev. Microbiol. 55: 165-199.

Morrison E.E. and J.A. Plumb. 1994. Olfactory organ of channel catfish as a site of experimental Edwardsiella ictaluri infection. J. Aquat. Anim. Health 6: 101-109.

Novak E.A., H. Shao, C.A. Daep and D.R. Demuth. 2010 Autoinducer-2 and QseC control biofilm formation and in vivo virulence of Aggregatibacter actinomycetemcomitans. Infect. Immun. 78: 2919-2926.

Parsek M.R. and E.P. Greenberg. 2005. Sociomicrobiology: the connections between quorum sensing and biofilms. Trends Microbiol. 13: 27-33.

Plummer P., O. Sahin, E. Burrough, R. Sippy, K. Mou, J. Rabenold, M. Yaeger and Q. Zhang. 2011. The critical role of LuxS in the virulence of Campylobacter jejuni in a guinea pig model of abortion. Infect. Immun. doi:10.1128/IAI.05766-11.

Rickard A.H., R.J. Palmer, D.S. Blehert, S.R. Campagna, M.F. Semmelhack, P.G. Egland, B.L. Bassler and P.E. Kolenbrander. 2006. Autoinducer 2: a concentration-dependent signal for mutualistic bacterial biofilm growth. Mol. Microbiol. 60: 1446-1456. Schauder S., K. Shokat, M.G. Surette and B.L. Bassler. 2001. The LuxS family of bacterial autoinducers: biosynthesis of a novel quorum-sensing signal molecule. Mol. Microbiol. 41: 463-476.

Sircili M.P., M. Walters, L.R. Trabulsi and V. Sperandio. 2004. Modulation of enteropathogenic Escherichia coli virulence by quorum sensing. Infect. Immun. 72: 2329-2337.

Sun K., H. Wang, M. Zhang, Z. Xiao and L. Su. 2009. Genetic mechanisms of multi-antimicrobial resistance in a pathogenic Edwardsiella tarda strain. Aquaculture 289: 134-139.

Surette M.G. and B.L. Bassle. 1999. Regulation of autoinducer production in Salmonella typhimurium. Mol. Microbiol. 31: 585-595.

Surette M.G., M.B. Miller and B.L. Bassler. 1999. Quorum sensing in Escherichia coli, Salmonella typhimurium, and Vibrio harveyi: a new family of genes responsible for autoinducer production. $P$. Natl. Acad. Sci. U.S.A. 96: 1639-1644.
Taga M.E., S.T. Miller and B.L. Bassler. 2003. Lsr-mediated transport and processing of AI-2 in Salmonella typhimurium. Mol. Microbiol. 50: 1411-1427.

Taga M.E., J.L. Semmelhack and B.L. Bassler. 2001. The LuxSdependent autoinducer Al-2 controls the expression of an ABC transporter that functions in Al-2 uptake in Salmonella typhimurium. Mol. Microbiol. 42: 777-793.

Thune R.L., D.H. Fernandez, J.L. Benoit, M. Kelly-Smith, M.L. Rogge, N.J. Booth, C.A. Landry and R.A. Bologna. 2007. Signature-tagged mutagenesis of Edwardsiella ictaluri identifies virulence-related genes, including a Salmonella pathogenicity island 2 class of type III secretion systems. Appl. Environ. Microb. 73: 7934-7946

Vendeville A., K. Winzer, K. Heurlier, C.M. Tang and K.R. Hardie. 2005. Making 'sense' of metabolism: autoinducer-2, LuxS and pathogenic bacteria. Nat. Rev. Microbiol. 3: 383-396.

Wagner B.A., D.J. Wise, L.H. Khoo and J.S. Terhune. 2002. The epidemiology of bacterial diseases in food-size channel catfish. J. Aquat. Anim. Health 14: 263-272.

Wang L., J. Li, J.C. March, J.J. Valdes and W.E. Bentley. 2005 Cyclic AMP (cAMP) and cAMP receptor protein influence both synthesis and uptake of extracellular autoinducer 2 in Escherichia coli. J. Bacteriol. 187: 2066-2076.

Waters C.M. and B.L. Bonnie. 2005. Quorum sensing: cell-to-cell communication in bacteria. Annu. Rev. Cell. Dev. Bi. 21: 319-346.

Williams M.L. and M.L. Lawrence. 2005. Identification and characterization of a two-component hemolysin from Edwardsiella ictaluri. Vet. Microbiol.108: 281-289.

Williams M.L., A.F. Gillaspy, D.W. Dyer, R.L. Thune, G.C. Waldbieser, S.C. Schuster, J. Gipson, J. Zaitshik, C. Landry, M.M. Banes and others. 2012. Genome sequence of Edwardsiella ictaluri 93-146, a strain associated with a natural channel catfish outbreak of enteric septicemia of catfish. J. Bacteriol. 194: 740-741.

Winzer K., K.R. Hardie, N. Burgess, N. Doherty, D. Kirke, M.T. Holden, R. Linforth, K.A. Cornell, A.J. Taylor, P.J. Hill and others. 2002. LuxS: its role in central metabolism and the in vitro synthesis of 4-hydroxy-5-methyl-3(2H)-furanone. Microbiology 148: 909-922.

Xavier K.B. and B.L. Bassler. 2005. Regulation of uptake and processing of the quorum-sensing autoinducer AI-2 in Escherichia coli. J. Bacteriol. 187, 238-248.

Xu L., H. Li, C. Vuong, V. Vadyvaloo, J. Wang, Y. Yao, M. Otto and Q. Gao. 2006. Role of the luxS quorum-sensing system in biofilm formation and virulence of Staphylococcus epidermidis. Infect. Immun. 74: 488-496.

Zhang M., K. Sun and L. Sun. 2008. Regulation of autoinducer 2 production and luxS expression in a pathogenic Edwardsiella tarda strain. Microbiol-SGM 154: 2060-2069.

Zheng W., Y. Hu and L. Sun. 2010. Cloning and analysis of a ferritin subunit from turbot (Scophthalmus maximus). Fish Shellfish Immun. 28: 829-836.

Zheng W. and L. Sun. 2011. Evaluation of housekeeping genes as references for quantitative real time RT-PCR analysis of gene expression in Japanese flounder (Paralichthys olivaceus). Fish Shellfish Immun. 30: 638-645. 